\title{
Test for Octupole Collectivity in Light Barium Isotopes
}

\author{
N. Minkov \\ Institute of Nuclear Research and Nuclear Energy, \\ Bulgarian Academy of Sciences, 72 Tzarigrad Road, 1784 Sofia, Bulgaria
}

Received 6 October 2021

doi: https://doi.org/10.55318/bgjp.2021.48.5-6.451

\begin{abstract}
This work explores the structure of yrast alternating-parity spectra of the light even-even Barium isotopes ${ }^{118-124} \mathrm{Ba}$ within a collective quadrupole-octupole rotation model (QORM). The model analysis of the band-structure and parity-shift effects in these nuclei indicates the persisting manifestation of octupole collectivity away from the double "octupole magicity" $(Z=56, N=88)$ and suggests that it may still govern the shape dynamics in the low Barium masses. On the other hand it is realized that different effects stemming from the intrinsic shell structure and the dominance of quadrupole deformation may have a competing role emphasizing on the need for further more detailed study of this mass region.
\end{abstract}

KEY WORDS: Quadrupole-octupole deformation, alternating-parity bands, nuclear collectivity, light Barium isotopes.

\section{Introduction}

The ${ }^{144} \mathrm{Ba}$ nucleus and the near Barium isotopes are of continuous interest in the nuclear structure study due to the octupole double-magic character of the proton and neutron numbers $Z=56$ and $N=88$ suggested by the shell model and associated with the appearance of strong octupole (reflection-asymmetric) correlations [1]. While in ${ }^{144,146} \mathrm{Ba}$ the octupole deformation was experimentally proven to exist even in the ground state [2], the possible evolution of the reflection asymmetry along the isotopic chain remains to be a subject of examination for various experimental and theoretical groups.

The objective of this work is to test the structure of the yrast positive- and negative-parity spectra, interpreted in terms of alternating-parity bands (APBs), in the much lighter isotopes ${ }^{118-124} \mathrm{Ba}$ for possible structure which can be associated with the manifestation of octupole collectivity. To this end we employ a 
collective quadrupole-octupole rotation model (QORM) approach [3,4] the application of which would clarify to what extent the alternating-parity spectra in ${ }^{118-124} \mathrm{Ba}$ carry the characteristics of the well recognized octupole bands observed in certain nuclear regions such as the light actinide nuclei [5]. Below it will be shown that the model analysis of the APB structure and, in particular, the displacement between opposite-parity energy levels (parity-shift) allows one to assess the stability (or the softness) of the octupole mode in the considered spectra compared to the well recognized cases and to evaluate on this basis the degree of octupole collectivity.

\section{QORM and Alternating-Parity Bands Structure}

QORM describes rotations of a quadrupole-octupole (QO) shape generated by a point-symmetry based Hamiltonian [3] together with low-energy oscillations in a double-well octupole potential [4]. The model provides an explanation of the APB structure typical for the nuclei with the presence of well pronounced (stable) QO deformation. This structure is characterized by a strong shift-up of the negative-parity levels with respect to the positive-parity ones at low angular momenta due to the strong penetration through the potential barrier and subsequent ordering of both sequences into a common "octupole" band at high angular momenta where the increased barrier suppresses the penetration and reduces the oscillation. So far the QORM was successfully applied in the study of octupole bands in Radium and Thorium isotopes [4] as well as in several Ba and Ce nuclei including ${ }^{144,146} \mathrm{Ba}[6]$. (For details on the model formalism and its application see [4].) Recently the model was applied to probe the octupole collectivity in light ${ }^{130-136} \mathrm{Nd}$ [7] showing a capability to indicate enhanced octupole collectivity with signs of a stable QO mode in ${ }^{136} \mathrm{Nd}$ [8] and softer QO degrees of freedom in the remaining isotopes. It was shown that the model gradually reaches the limiting case of a transition between rigid and soft $\mathrm{QO}$ modes with the latter being described by another version called coherent quadrupoleoctupole model (CQOM) $[9,10]$.

Taking stock of the above experience in the present work we consider that using the "rigid" QORM only will suffice to assess the overall octupole capability of the light Barium isotopes of interest. The assessment is quantitatively enhanced by the use of the so-called "staggering" analysis in which the following fivepoint finite-differences expression is applied to the APB energy $E$ as a function of the angular momentum $I$ :

$$
\operatorname{Stg}(I)=6 \Delta E(I)-4 \Delta E(I-1)-4 \Delta E(I+1)+\Delta E(I+2)+\Delta E(I-2),
$$

where $\Delta E(I)=E(I+1)-E(I)$. It has been shown that in the higher angularmomentum regions of the octupole bands in light actinide nuclei (Rn, Ra, Th), where stabilized QO shapes are considered, the quantity (1) reveals the so-called "beat" staggering structure [5] of the spectrum. This effect has been explained 
Test for Octupole Collectivity in Light Barium Isotopes

through the high-order angular momentum terms in the QORM Hamiltonian appearing as the result of a specific point-symmetry based property of the rotating QO shape. The same was shown to manifest also in ${ }^{144,146} \mathrm{Ba}$ isotopes [6]. Aside from the ultimate cases of well pronounced (stable) QO deformation the high-order derivative carries useful information about the evolution of the parityshift effects and the development of the deformation mode along the APB [6,7]. Namely in this aspect its application is of use in the analysis given below.

\section{Alternating-Parity Bands in ${ }^{118-124} \mathrm{Ba}$}

The QORM has been applied to the APBs in the four even-even ${ }^{118-124} \mathrm{Ba}$ isotopes by adjusting the set of model parameters (see [4] for details on parameters) to the experimental yrast positive- and negative-parity levels [11] up to angular momentum $I=20$. In this way all considered spectra are described on the same footing being limited below the second backbending region.

The theoretical and experimental APB structures are compared in Figure. 1. First of all we see that the theoretical patterns well reproduce the angular momentum path of the experimental spectra in all four isotopes. The corresponding rootmean-square deviations between theory and experiment vary between 65 and 84
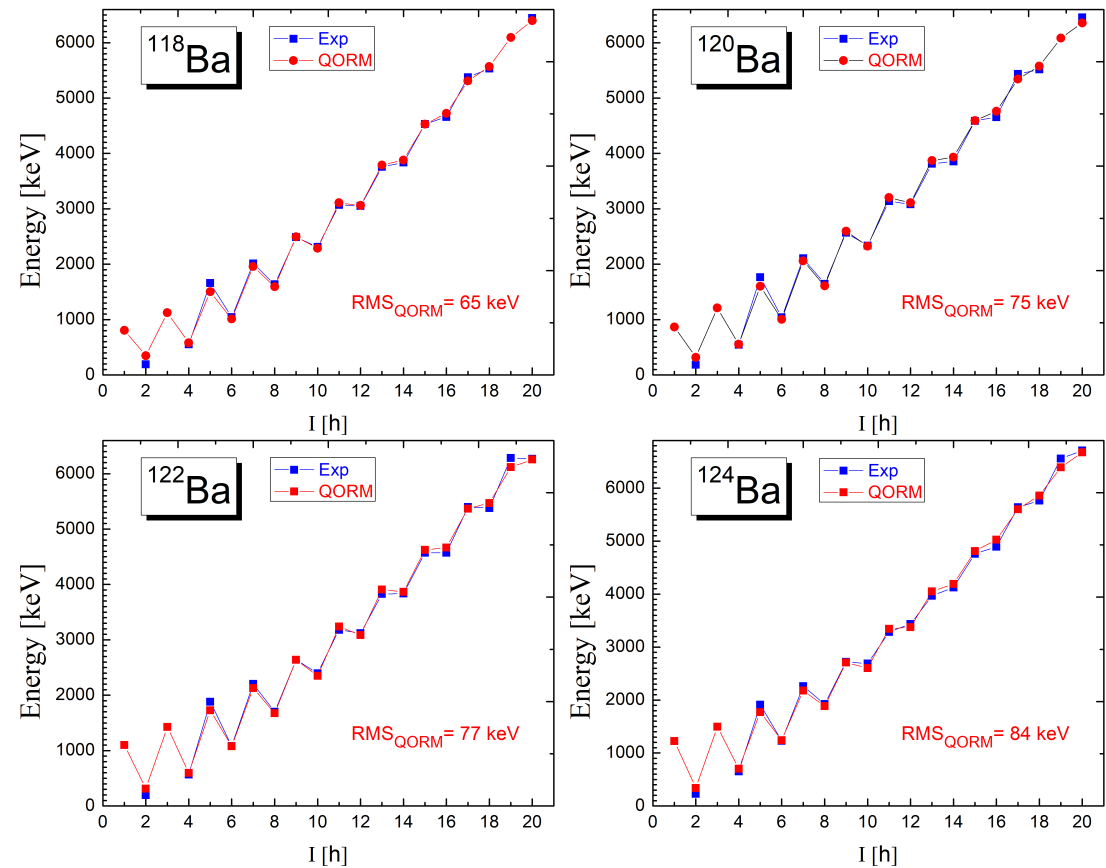

Figure 1. (Color online) QORM description of APBs in ${ }^{118-124} \mathrm{Ba}$. 
$\mathrm{keV}$ allowing a reasonable quantitative assessment of the octupole ability of the considered nuclei in terms of the model approach. The parameters of the fits are not given for simplicity and are available from the author upon request. Here we can only say that the obtained values are in the ranges typical for the other applications of the model [4, 6-8].

The more careful look at the higher-spin part of the spectra (both theory and experiment) shows that in ${ }^{118} \mathrm{Ba}$ and ${ }^{124} \mathrm{Ba}$ the opposite-parity counterparts closer approach each other compared to the other two isotopes. In terms of the model interpretation this means that the octupole oscillations in the double-well potential are more strongly suppressed and both APBs tend to form corresponding octupole bands. The observed behaviour of the energy spectra can be then qualified as a sign of octupole collectivity.

The above picture is better illustrated in terms of the odd-even staggering patterns obtained by applying formula (1) to each of the studied APBs. The result is given in Figure 2. Now we clearer see the stronger decrease of the staggering amplitude in ${ }^{118} \mathrm{Ba}$ and ${ }^{124} \mathrm{Ba}$. Furthermore, the finer staggering patterns reveal in both spectra an angular momentum region of $I=13-15$ where the amplitude reaches a minimum after which a slight increase is observed resembling the beats in the staggering patterns of octupole bands. Of course, here the latter is
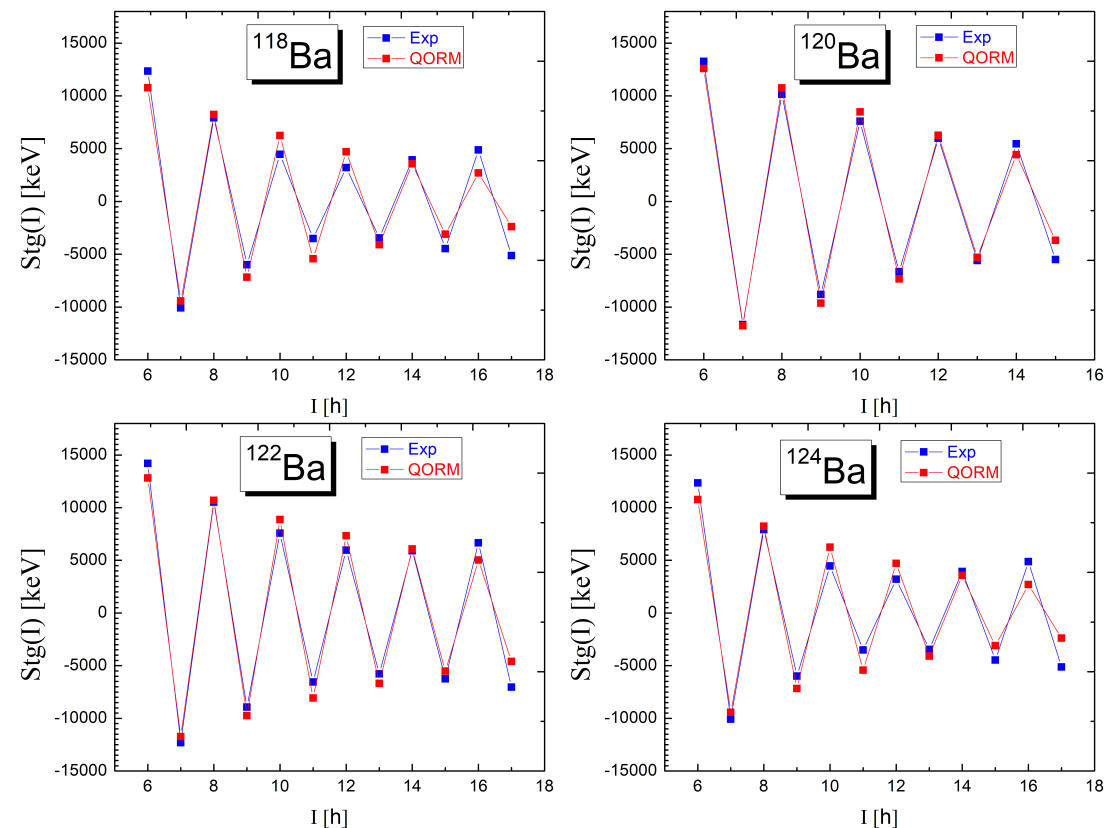

Figure 2. (Color online) Experimental and theoretical staggering patterns for the APBs in ${ }^{118-124} \mathrm{Ba}$. 
Test for Octupole Collectivity in Light Barium Isotopes

not the case since the parity-shift does not reach zero value and does not change in sign. We, therefore, can only speak about signs of octupole collectivity rather than about its manifestation.

Comparing the present results for ${ }^{118-124} \mathrm{Ba}$ with the analysis made in ${ }^{130-136} \mathrm{Nd}$ [7] we remark that the structure of all four Barium APBs is rather similar to that in ${ }^{134} \mathrm{Nd}$ which was considered in the high-spin part to be on the border between the stable and soft QO deformation modes. We can, therefore, further specify that our current model analysis indicates signs of intermediate (between stable and soft) octupole collectivity in the region of light Barium isotopes.

At this point, however, we should recognize that no definite conclusion about the role of the octupole mode can be made isolated from other possible degrees of freedom. It is known that in the overall spectra of the considered nuclei there are indications for a rich diversity of quasi-particle excitations with superposed rotational bands and simultaneous manifestation of quadrupole triaxiality [12]. In addition, a detailed analysis of the E1 and E2 transition rates in the APBs is needed in order to reach more confident estimates about the role and competitiveness of the reflection-asymmetric modes in the collective dynamics of the Barium nuclei away from the octupole double-magic point.

\section{Concluding Remarks}

In conclusion, the simple QORM description of the APBs in the light Barium isotopes reveals persisting signs of octupole collectivity identified on the border between stable and soft octupole deformation. This may be considered as a residual of the double octupole magicity which evolves along the Ba isotopic chain keeping the effect of the single $Z=56$ octupole magic number. The obtained result is consistent with analyses made in other nuclei, such as the light Nd isotopes, and points out the possible more global role of the reflectionasymmetric degrees of freedom in the overall nuclear region. However, further more detailed theoretical and experimental study involving other competing degrees of freedom and deformation modes is necessary in order to reach more definite conclusion.

\section{Acknowledgements}

This work is supported by the Bulgarian National Science Fund (BNSF) under Contract No. KP-06-N48/1. The author thanks Prof. Costel Petrache for the illuminating discussions on the experimental data and structure of light Barium isotopes. 


\section{N. Minkov}

\section{References}

[1] P.A. Butler and W. Nazarewicz (1996) Rev. Mod. Phys. 68349.

[2] B. Bucher et al. (2016) Phys. Rev. Lett. 116 112503; (2017) Phys. Rev. Lett. 118 152504.

[3] N. Minkov, S. Drenska, P. Raychev, R. Roussev and D. Bonatsos (2001) Phys. Rev. C 63044305.

[4] N. Minkov, P. Yotov, S. Drenska, W. Scheid (2006) J. Phys. G: Nucl. Part. Phys. 32 497.

[5] D. Bonatsos, C. Daskaloyannis, S. Drenska, N. Karoussos, N. Minkov, P. Raychev and R. Roussev (2000) Phys. Rev. C 62024301.

[6] N. Minkov, S. Drenska and P. Yotov, Nuclear Theory, vol. 35, Proc. of 35-th Int. Workshop on Nuclear Theory (Rila, Bulgaria 2016), ed. M. Gaidarov and N. Minkov, (Heron Press, Sofia), p. 244 (2016) [http://ntl.inrne.bas.bg/workshop/2016/contributions/a25_Minkov_2016.pdf].

[7] N. Minkov (2019) Bulg. J. Phys. 46 (No 4) 386.

[8] C.M. Petrache, N. Minkov, T. Nakatsukasa, et al. (2020) Phys. Rev. C 102014311.

[9] N. Minkov, P. Yotov, S. Drenska, W. Scheid, D. Bonatsos, D. Lenis and D. Petrellis (2006) Phys. Rev. C 73044315.

[10] N. Minkov, S. Drenska, M. Strecker, W. Scheid and H. Lenske (2012) Phys. Rev. C 85034306.

[11] http://www.nndc.bnl.gov/ensdf/.

[12] C.M. Petrache, private communication (2021). 